\title{
High-SPEed Image Processing TeChNiQue IMPLEMENTATION FOR POINTING AND TRACKING SYSTEM ENABLING Free-SPACE OPTICAL COMMUNiCATIONS
}

\author{
Daria Stepanova, Valentin Pryanichnikov, Sergey Khandorin, \\ Alexey Kuznetsov \& Andrey Kulchitsky
}
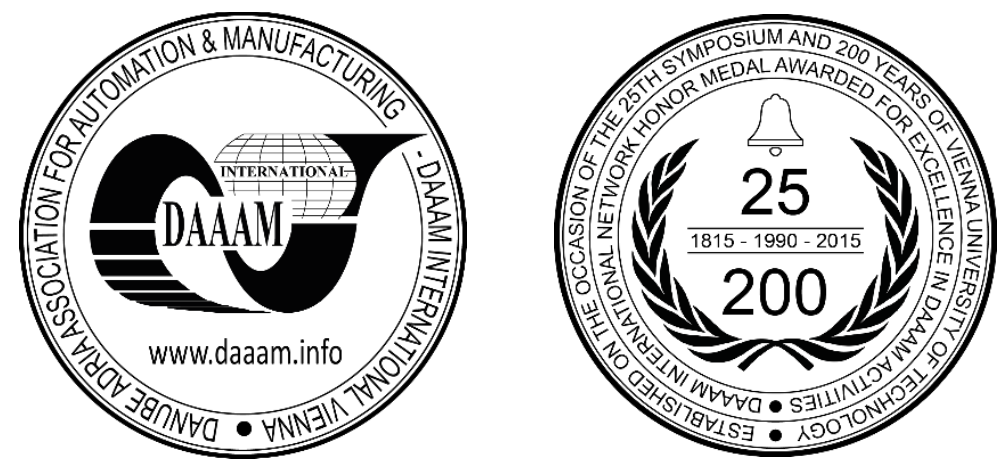

This Publication has to be referred as: Stepanova, D[aria]; Pryanichnikov, V[alentin] E.; Khandorin, S[ergey]; Kuznetsov, A[lexandr] \& Koulchitskiy, A[ndrey] (2018). High-speed Image Processing Technique Implementation for Pointing and Tracking System Enabling Free-Space Optical Communications, Proceedings of the 29th DAAAM International Symposium, pp.1306-1312, B. Katalinic (Ed.), Published by DAAAM International, ISBN 978-3-90273420-4, ISSN 1726-9679, Vienna, Austria

DOI: $10.2507 / 29$ th.daaam.proceedings.188

\begin{abstract}
Free-space laser communication technology has several advantages comparing with radio communications for various applications on Earth and in space. An optical transmission line can be characterized by higher data rates in combination with lower power consumption and lower weight of the communication terminal, which is an undoubted advantage for using onboard satellites, space telescopes, and scientific space probes. The main challenge of implementing laser communication between various mobile platforms is based on the need to use a precise and highspeed controller for a pointing and tracking system and the visual system, which shall operate at frequencies significantly higher than frequencies of a standard television sweep.This work is dedicated to the development of a pointing and tracking system for an optical communication terminal for different configurations of mobile platforms: satellites, mobile robots, and unmanned aerial vehicles. Present work is based on derived earlier requirements for each of these configurations. During present work, hardware for the optical terminal development was selected and control algorithms were created for pointing and tracking drives. The image-processing algorithm was tested to generate the signal for the control system represented by the PID controller. An algorithm is based on the idea of detecting the center of an image from the orientation beacon received by the video sensor of the optical communication terminal. FPGAbased implementation of the image-processing algorithm reduced drive control latency, which in turn improved the accuracy of the pointing and tracking system and increased the efficiency of data transmission.
\end{abstract}

Keywords: free-space optical communications; image processing; pointing and tracking algorithms; mobile robots

\section{Introduction}

Free-space optical communication has become attractive in recent years due to various benefits compared to radio communications: higher data rate, lower mass and smaller terminal size. There are numerous projects dedicated to the development of laser communication capabilities for satellites, unmanned aerial vehicles (UAV) and mobile robots. 
For example, several projects of laser link establishment on low earth orbit (LEO) and Geostationary orbit (GEO) have already shown data rates up to $4.6 \mathrm{Gbps}$ and communication distance of thousands of kilometers [1-4]. There are several projects of gyro-stabilized platforms for UAVs optical communications in the stage of the development [5-8]. Mobile robots carrying optical communication payloads found applications for operations in hazardous environments [9-11].

In order to provide a stable communication link between mobile platforms taking into account the narrowness of a laser beam, it is important to achieve a highly accurate beam acquisition, pointing and tracking. Thus, all projects addressing the need for establishing a free-space optical are facing a challenge of developing a precise pointing, acquisition, and tracking (PAT) system. The link establishment classical approach is based on laser beam spot detection by highly sensitive sensors matrix, embedded into a reception optical system, and calculation of its center [12-13]. In other words, a collimated laser beam from one communication node passes through lens systems and forms a spot image on the optical detector of another communication node. The measured position of the spot is converted into the direction of a beam. Based on deviation from the reference beam direction PAT controller generates the signal for terminal drives.

A standard PAT system architecture consists of two components: coarse pointing and fine pointing and utilizes separated sets of sensors and drives for these stages [14]. On the coarse pointing stage, after the application of spatial filter and threshold comparison, spot-center coordinates are calculated to provide the correct angle for drives. At this stage, most of the image-processing algorithms analyze up to 200 frames per second [15]. The fine pointing stage normally utilizes fast steering mirrors and sensors that are able to feed the controller with a sample rate of more than 1000 frames per second [16]. Combination of two components of a PAT system is normally increasing the optical terminal size and weight due to a complex optical system: most of the proven terminals weight more than $30 \mathrm{~kg}$. This mass might be critical for applications on small satellites, mobile robots and UAVs lighter than $50 \mathrm{~kg}$.

This work proposes a new design approach for the PAT system development, which considerably decreases the mass and size of a laser terminal and provides required pointing accuracy. The overall PAT architecture is described in Chapter 2. The work addresses the question of advancing a laser beam processing technique and its implementation to combine coarse and fine pointing systems in one and thus simplify the control system. Chapter 3 provides an overview of control strategy simplification for PAT systems, while image processing technique and its implementation are presented in Chapter 4. The specifications of system evaluation are outlined and the performance of both systems is evaluated and compared in Chapter 5.

\section{System architecture}

A laser terminal is divided into two systems: optical and PAT systems. The laser system includes incoming and outcoming beam processing stages: collimation, signal modulation, and demodulation and data retrieval. A PAT system is presented by a gimbal with two angles of freedom and consists of motors, their drives and sensors set. PAT motors control the orientation of the whole optical terminal in two angles: pitch and yaw. An optical subsystem is designed to minimize size and weight of a moving part. PAT feedback sensors allow the precise determination of rotation of motors. Laser communication terminal shown on Fig. 1 works as follows. A laser beacon from partner platform arrives in the optical system and PAT telescope. Laser receiver module retrieves the payload data and sends it to the main computer.

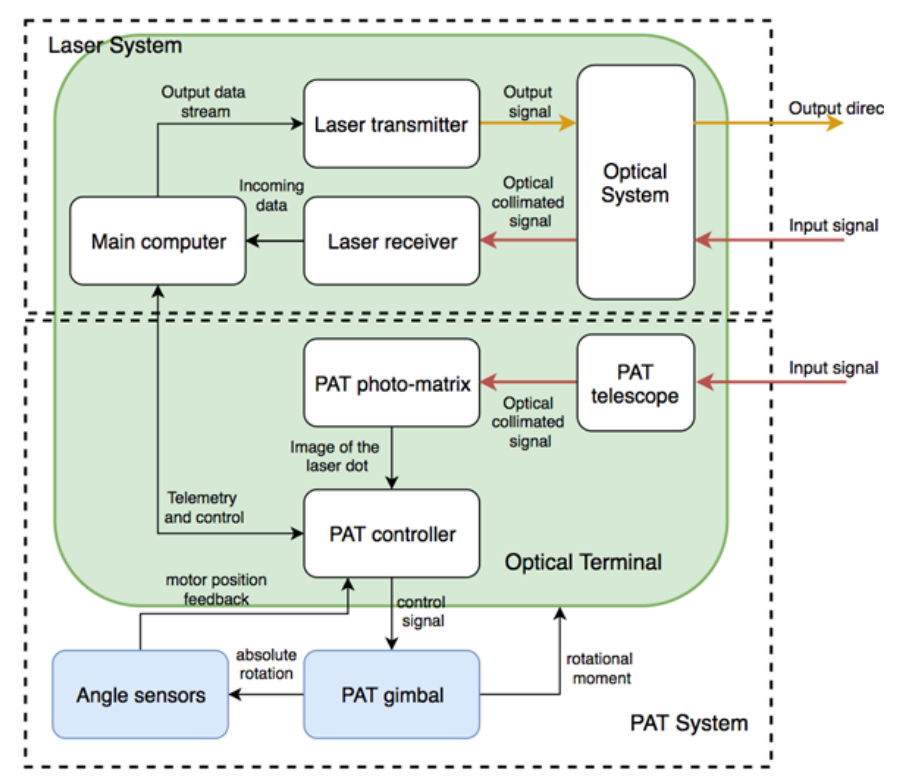

Fig. 1. Laser terminal system architecture 
PAT system, based on the same processed signal, corrects the pitch and yaw angles of the terminal, thereby setting the direction of propagation of the outgoing optical beam. The main computer sets the initial guidance angles for the control system and generates data for transmission.

\section{Control Strategy}

The task of the drive system control algorithm is to ensure accurate pointing of the laser module towards a partner platform. For all three stages of pointing, acquisition, and tracking, a PID algorithm was chosen due to its quick setup and testing. Control for each coordinate is carried out independently. A block diagram of the gimbals control system is given in Fig. 2.

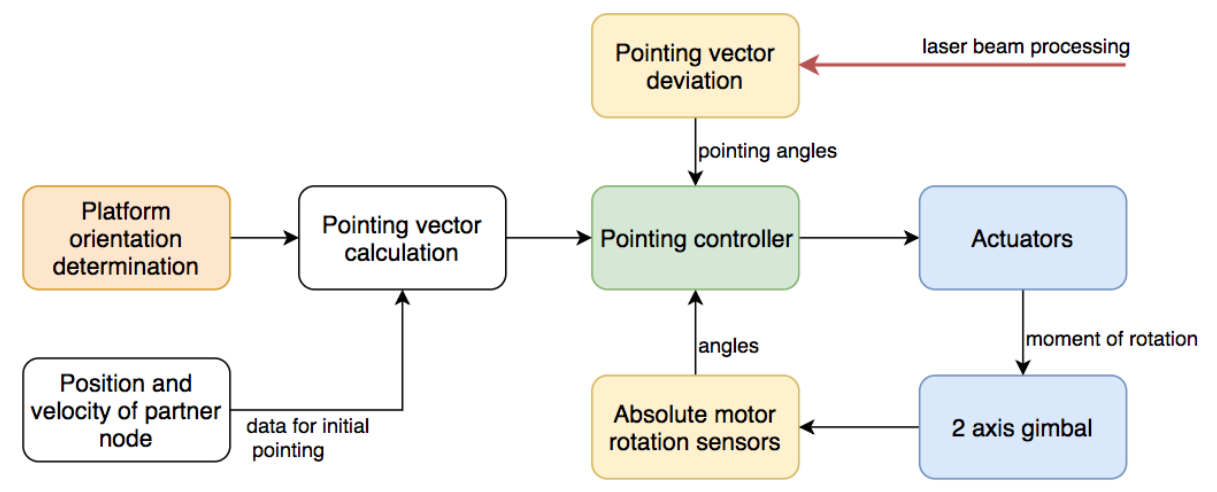

Fig. 2. PAT control system high-level diagram

It is assumed, that at each moment of time, the communication node estimates its orientation and position and also has sufficient information about the position and velocity vector of a partner node. Thus, with the reference position and angular rate, a pointing vector is calculated. The design uses the drive sensors, implemented in the form of a sine-cosine rotating matrix, and the deviation angle calculated by the video sensor as data sources about the current position of the motors. Based on the data obtained, the deviation of the current position of the drive from the required is calculated, and the information from the two sensors is reduced to one scalar value using the coefficients set at the time of calibration of the device. During the initial search for a signal, when information from the video sensor is not available, only data from the platform orientation and partner position propagator is used. Data is fed to the PID part of pointing controller, which derives the control action. The pointing controller also includes the compensator of friction, which allows smooth control at slow angular rates. The control law is described by the following formula:

$$
A_{Y}(t)=K_{p} e(t)+K_{i} \int_{0}^{t} e(t) d t+K_{d} \frac{d e(t)}{d t}
$$

where $A_{Y}(t)$ - control action, e(t) - angle error, $K_{p}, K_{i}$ and $K_{d}$ - coefficients of PID regulator. The center of the image along the pitch angle is calculated in a similar way.

\section{Image processing algorithm description}

The choice of the image processing algorithm and its implementation is critical for correct PAT system behavior due to the influence of delay between measurements and processing quality. The current system was built to minimize the latency of the control circuit making the system sufficiently stable and still using the image sensor of high resolution. Implementing an algorithm directly on the sensor board eliminated additional delays in image transfer through various interfaces between PAT subsystems.

The main goal of the video sensor processing algorithm is to determine the center of the image of the orientation beacon. Image processing here can be done line-by-line eliminating the need of storing the whole image in the memory. For each row, the coordinate of row center is calculated as the weighted sum of the numbers of the pixels. The weighted sum of the obtained line centers is then calculated along the $\mathrm{x}$ and $\mathrm{y}$ coordinates. Let it be the number of a pixel $(0 \leq \mathrm{i}<\mathrm{N})$ and $V_{i}$-its brightness, $0 \leq j \leq i, 0 \leq k \leq j$. The center of the image on the one-dimensional line can be found using two accumulating adders $\mathrm{A}$ and $\mathrm{B}$, where $B_{N-1}$ is a weighted sum of reverse pixel numbers $(N-1-j)$, and $A_{N-1}$ is a sum of pixel brightnesses:

$$
A_{i}=\sum_{j=0}^{i} V_{j}
$$




$$
B_{i}=\sum_{j=0}^{i} A_{j}=\sum_{j=0}^{i} \sum_{k=0}^{j} V_{k}=\sum_{j=0}^{i} V_{j}(i-j)
$$

Therefore, center of an image can be found as:

$$
x_{i}=\frac{B_{N-1}}{A_{N-1}}=\frac{\sum_{j=0}^{N-1} V_{j}(N-1-j)}{\sum_{j=0}^{N-1} V_{j}}
$$

The center of the image along the other axis is calculated in a similar way.

\section{Algorithm implementation and results}

The first implementation of the control algorithm together with an image processing algorithm was made in Matlab. After that, the test setup for system and algorithm validation was developed. Test setup included a control system implementation on the external microcontroller, while an image processing technique was still performed on the PC. The second system implementation was done with the use of a Field Programmable Gate Array (FPGA), which was chosen for its high-speed processing capability and low power consumption.

\section{Case 1: PAT system prototyping for mobile robots communication}

The choice of hardware for the configuration of mobile robots is based on a combination of its low cost and the minimum required technical characteristics for the purpose of rapid prototyping and algorithms testing. Thus, Arduino and BeagleBone development boards were chosen as computational modules. Brushless motors were chosen as the drives for the PAT system, COTS sensors 9DOF (degrees of freedom) and potentiometers were used for the feedback sensor system. Control system for brushless motors was implemented on the Atmel SAM3X8E ARM Cortex-M3 CPU that is a part of Arduino due board.

As for image sensor, an HD-Wide-Angle Camera Module from Raspberry pie was used. A camera module aims to deliver up to 90 frames per second with 5-megapixel resolution. Electrical and optical systems were fixed on the ABS manufactured structure. Motors were capable of rotating the body of the terminal in $\pm 90^{\circ}$ in pitch angle as well as $\pm 90^{\circ}$ in yaw angle. Error! Reference source not found. and Error! Reference source not found. demonstrate the receiver module prototype and experimental setup for system validation.

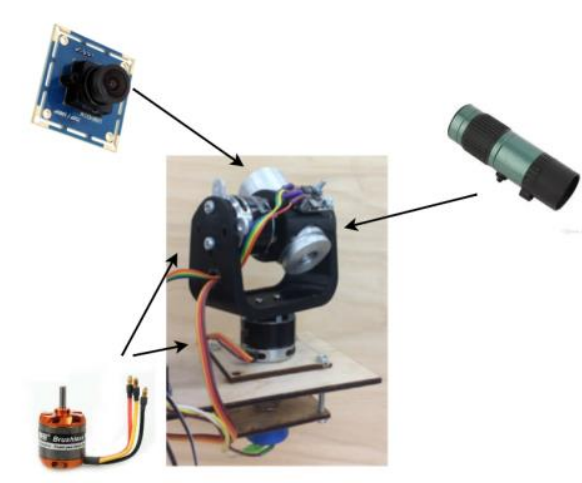

Fig. 3. Receiver module implementation

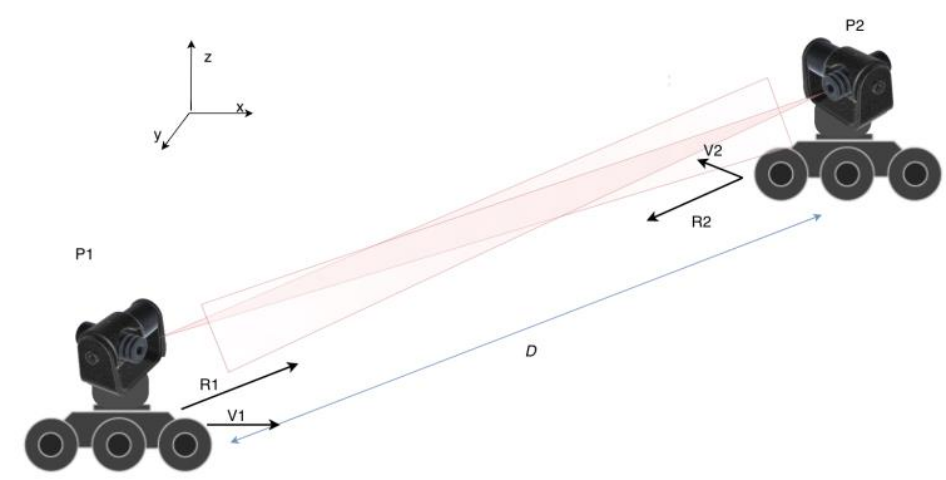

Fig. 4. Experimental setup

To validate the system design and algorithms choice, a working prototype was developed and tested. The test bed consisted of 2 sets of transmission and reception modules, installed on mobile platforms. During the test, platforms were moving relative to each other adopting their pointing angle by described above algorithm.

The mean distance between platforms during the test was $10 \mathrm{~m}$, varying from 1 to $15 \mathrm{~m}$. Power of the transmitter was set to $1 \mathrm{~W}$ with beam divergence varying from $1 \mathrm{deg}$ to $6 \mathrm{deg}$. The mean velocity of each mobile platform was varying from $1 \mathrm{~cm} / \mathrm{s}$ to $5 \mathrm{~cm} / \mathrm{s}$.

Error! Reference source not found., Error! Reference source not found. and Error! Reference source not found. visualize the image processing algorithm utilization during the test campaign from detection of the laser spot, its center, and determination of a direction of partner communication node. 


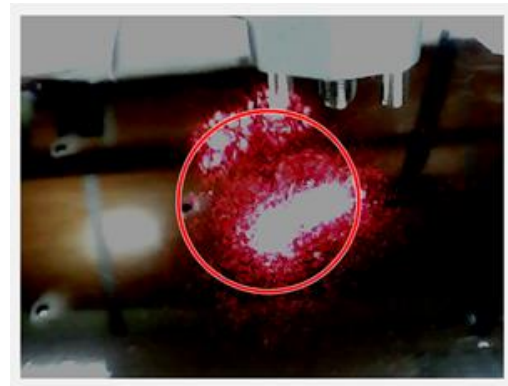

Fig. 5. Image acquisition

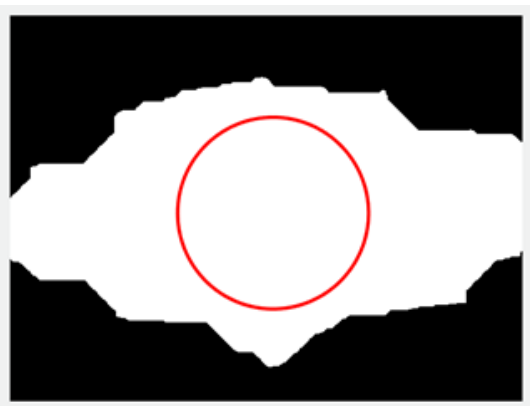

Fig. 6. Example of laser beam spot processing

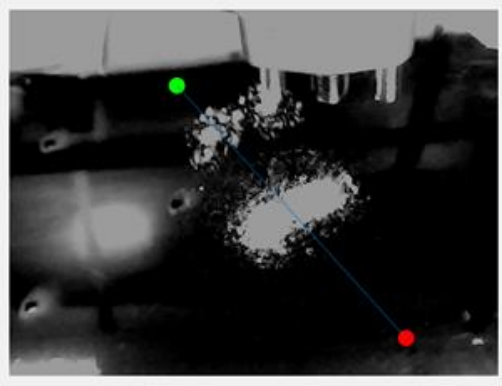

Fig. 7. Example of direction determination

Several sets of tests were conducted with different trajectories of mobile platforms. During tests, an accuracy of pointing error estimation with image processing algorithm was evaluated depending on different relative angular rates of platforms and Fig. 8 shows an example of their dependency. Pointing error was calculated by described in Chapter 4 algorithm; the angular rate was calculated based on mobile platform trajectories.

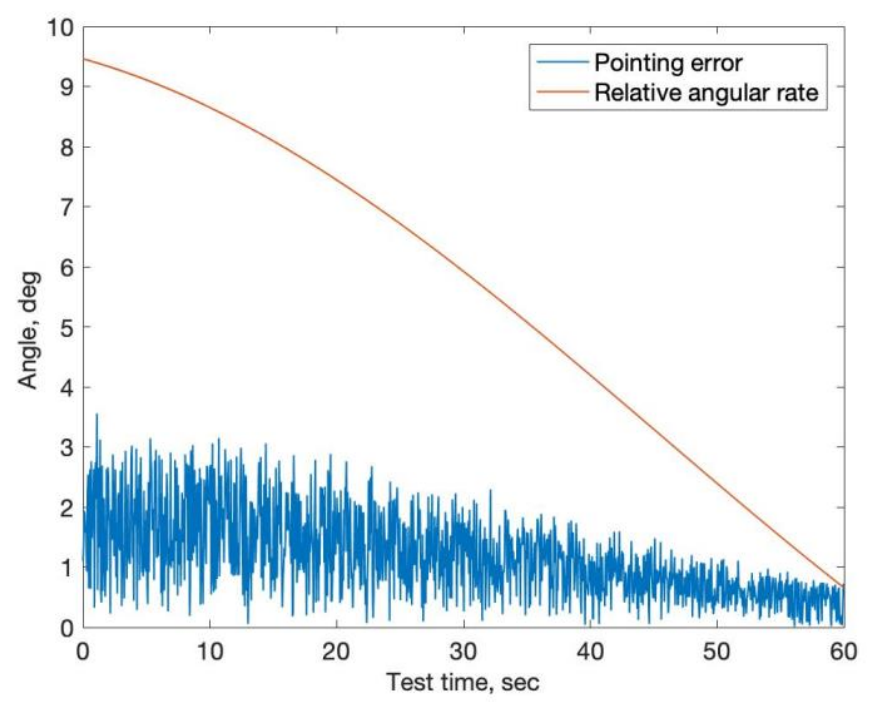

Fig. 8. Pointing error dependence on relative angular rate

It can be observed, that pointing error line is fluctuating - this is due to a vibrational environment of a mobile platform, where the laser terminal was installed. PID controller was later eliminating the high-frequency component for the control signal generation. During the test, pointing error did not exceed $3 \mathrm{deg}$ with maximum relative angular rate $12 \mathrm{deg} / \mathrm{s}$. Control system latency has achieved $26 \mathrm{~Hz}$. The main source of delay was an image processing algorithm and interface between the platform and PC. Thus, with the developed setup, algorithm and system design were validated.

\section{Case 2: PAT system prototyping for satellite communication}

The next step of laser terminal development and proof of design and algorithms feasibility was to select the hardware for satellite PAT prototyping and implement the image-processing algorithm on the FPGA. The choice of hardware for the satellite-to-satellite configuration is based on the need to ensure the high reliability of the apparatus being developed in low-Earth orbit conditions. A flash-based FPGA immune to firm errors was selected for the implementation of the laser terminal for the satellite configuration. The PAT module was developed as one unit: PAT drives were fixed on a base, which can possibly be attached to the spacecraft, providing a tilt for the optical mobile unit by $\pm 10^{\circ}$ in pitch angle and $\pm 30^{\circ}$ in yaw angle.

Electronics of PAT system, which processes information from the sensors of yaw and pitch, as well as images from a video camera, and on the basis of this data, generates control actions on the engines, is distributed between PAT submodules. The board of the angle sensor with a CMOS sensor and FPGA is installed on the movable module, the rest of the board - at the base of the device. The optical system is connected directly to the collimators using a pair of optical waveguides, bypassing the PAT system. This provides additional flexibility for the design of an optical system and independent testing of two systems. 
As for image sensor, VITA 1300 (NOIV1SN1300A) by ON Semiconductor was used. It transmits data over four LVDS lanes running at 620 Mbps. A Smartfusion 2 (M2S090-1FCS325) FPGA by Microsemi is used to interface a sensor and is located on the other side of the sensor board. The FPGA performs the image processing by itself and transmits only computed coordinates, which is a small amount of data, to the main control system board. Storage of a full resolution image would require at least 10 Mbits of memory, which exceeds the amount of internal memory available in the FPGA. The algorithm implementation performs computation on the fly and avoids the need to store the full image in memory.
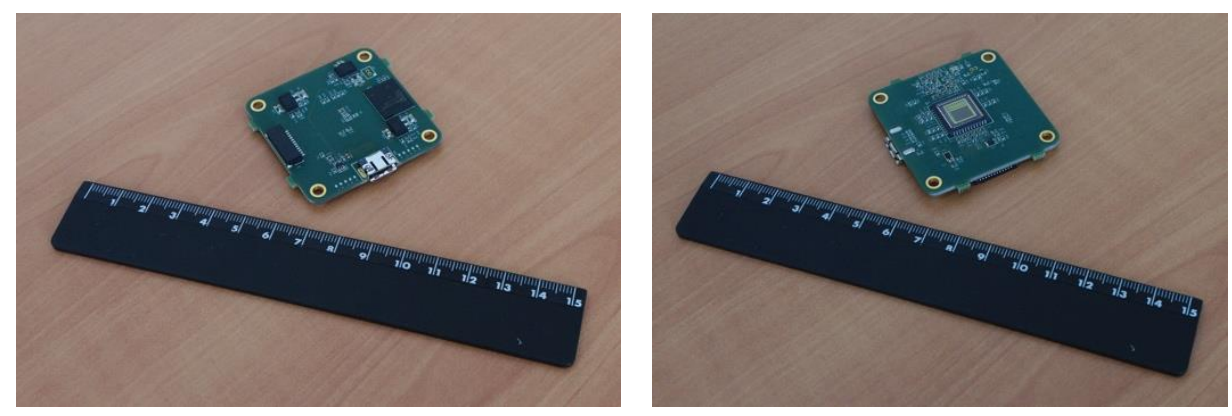

Fig. 9. Receiver module implementation for satellite scenario

The laser beam algorithm implementation on FPGA is currently in process. Based on the estimation, high pointing accuracy is feasible to achieve capturing 150 full-resolution frames per second. Tracking accuracy can be supported by utilization of partial readout feature, which will allow the selection of a subset of the image area thus increasing rate to 1650 frames per second. Thus, target accuracy of the system in development is set to 10 ',

\section{Conclusion}

This work has demonstrated the evolution of PAT system development for free-space optical communications for different platforms. Developed algorithms for the control system including the image processing technique proved their feasibility and applicability for mobile robots communication and showed the potential for satellite case scenario. Testing laser terminals on mobile robots validated the general system concept, algorithms selection, and control system design. The mean control accuracy during a test achieved 2 degrees, control system frequency was maximized to $26 \mathrm{~Hz}$. FPGA implementation of an image-processing algorithm, which can possibly increase the control loop frequency to $1650 \mathrm{~Hz}$, has started with a choice of hardware. Based on our estimates, we expect the system to achieve at least 10" pointing accuracy.

For future work, first of all, image processing algorithm implementation shall be completed and tested. Besides that, pointing system control loop can be further improved. For that, methodology for a model definition for a system of 2 axis gimbal and high-speed photo-sensor shall be developed. After that, the model of PAT shall be defined and validated. Later, the advanced control technique (Hinf, Adaptive controller) can be possibly implemented. Besides that, the image-processing algorithm can also be improved by noises mitigation.

\section{Acknowledgments}

The paper was partially supported by RFBR, projects 16-07-01264, 16-07-00935.

\section{References}

[1] V. W. S. Chan, “Free-space optical communications,” J. Lightwave Tech., vol. 24, no. 12, pp. 4750-4762, 2006.

[2] Gilles Planche, Vincent Chorvalli, SILEX in-orbit performances, Proceedings of the 5th International Conference on Space Optics, Toulouse, France, 30 March - 2 April, 2004.

[3] Nguyen, Tam, et al. "Development of a pointing, acquisition, and tracking system for a CubeSat optical communication module." SPIE LASE. International Society for Optics and Photonics, 2015.

[4] Keizo Nakagawa, Akio Yamamoto,"Pleminary design of LaserUtilizing Communications equipment (LUCE) installed on Optical inter-orbit communications engineering test satellite", National Space Development Agency of Japan (NASDA)

[5] Mynaric. (2018). Air Terminals. [online] Available at: https://mynaric.com/products/air/ [Accessed 24 Oct. 2018 ].

[6] Projecthyperion.co.uk. (2018). Hyperion Project. [online] Available at: http://projecthyperion.co.uk/ [Accessed 24 Oct. 2018].

[7] Sofka, Josef, Vladimir V. Nikulin, Victor A. Skormin, David H. Hughes, and David J. Legare. "Laser communication between mobile platforms." IEEE Transactions on Aerospace and Electronic Systems 45, no. 1 (2009): 336-346. 
[8] Majumdar, Arun K. "Free-space laser communication performance in the atmospheric channel." Journal of Optical and Fiber Communications Reports 2, no. 4 (2005): 345-396.

[9] Arnold, Robert, Richard G. Trissel, and Scott Bloom. "Free-space laser communication system having six axes of movement." U.S. Patent 6,347,001, issued February 12, 2002.

[10] Pryanichnikov, Valentin, and Daria Stepanova. "Laser Communication Prototyping For The Group Of Mobile Robots." Annals of DAAAM \& Proceedings 27 (2016).

[11] Kerr, D., K. Bouazza-Marouf, K. Girach, and T. West. "Free space laser communication links for short range control of mobile robots using active pointing and tracking techniques." (1996): 11-11.

[12] N. Perlot, M. Knapek, D. Giggenbach, J. Horwath, M. Brechtelsbauer, Y. Takayama, T. Jono, "Results of the optical downlink experiment KIODO from OICETS satellite to optical ground station oberpfaffenhofen (OGSOP)," in Proceedings of SPIE, vol. 6457, 2007

[13] Timothy, LaMar K., Jeffrey S. Sato, Alan M. Buchanan, and Patricia F. Batzer. "System and method for pointing and control of an antenna." U.S. Patent 7,333,064, issued February 19, 2008.

[14] Rybak, S. C., S. I. Lieberman, L. L. Hartter, R. L. Gregory, A. K. Nakashima, and R. F. Kaczynski. "Achieving ultrahigh accuracy with a body pointing CMG/RW control system." (1973).

[15] T.S. Rose, S.W. Janson, S. LaLumondiere, N. Werner, D.H. Hinkley, D.W. Rowen, R.A. Fields and R.P. Welle, "LEO to ground optical communications from a small satellite platform", The Aerospace Corporation

[16] Miyatake, Katsumasa, Yuta Fujii, Masaki Haruna, Jiro Suzuki, Kazuhide Kodeki, Shiro Yamakawa, and Tatsuyuki Hanada. "Development of acquisition and tracking sensor for next-generation optical inter-satellite communication." In Space Optical Systems and Applications (ICSOS), 2011 International Conference on, pp. 132135. IEEE, 2011. 